\title{
The International Pharmaceutical
} Federation publishes an overview of models for reporting medicine shortages

\author{
Stephanie Kohl
}

The International Pharmaceutical Federation (FIP) has published an overview of models for reporting medicine shortages. The report, which is intended to support the implementation of the World Health Assembly Resolution on "Addressing the global shortage of medicines and vaccines", indicates that globally medicine shortages have worsened.

The analysis underlying the report involves systems operated by Australia, Canada, the Netherlands, South Africa, Slovakia and the United States. It compares and contrasts features such as the scope of the shortages listed, the criteria applied (such as the predicted duration of a shortage) and the sources of information used by the eight different reporting systems. Of the six countries Slovakia and the United States are both currently operating two separate systems.

By sharing best practices the report aims, on the one hand, to guide the development and improvement of reporting shortages in countries that have a system in place and also in countries which are currently developing one. On the other hand, it raises awareness of the importance of detecting and understanding the causes of medicine shortages worldwide.

\section{EIU: REPORT ON CANCER MEDICINE SHORTAGES}

The Economist Intelligence Unit (EIU) published a report on cancer medicine shortages in Europe, which discusses the causes of shortages and provides policy recommendations. The study underlying the report was supported by the European Society for Medical Oncology (ESMO).

Patient care is significantly affected by medicine shortages as they increase the potential for error or adverse events, lead to the use of less desirable alternative products or even to the failure of, or delay in, treatment. Thus, EIU was

Stephanie Kohl, Department of Policy and Advocacy, European Association of Hospital Pharmacists, Rue Abbé Cuypers 3, Brussels 1040, Belgium; Stephanie. Kohl@eahp.eu commissioned by ESMO to investigate the impact of cancer medicine shortages in Europe.

The research team reviewed academic and grey literature to identify the effect of medicine shortages on the provision of care and ways to alleviate or prevent them. In addition, the research team discussed selected policy initiatives with a panel of experts. The European Association of Hospital Pharmacists' (EAHP) immediate past president Roberto Frontini was part of the expert panel.

One of the studies used for the development of the policy recommendations was EAHP's survey of medicine shortages, which listed cancer drugs as most likely to be affected. The report proposes the following policy recommendations:

- introduce legislation for early notification requirements for medicine shortages;

- establish strategic plans for medicine shortages;

- develop catalogues of shortages;

- develop essential medicines lists and assess the risk for shortages;

- introduce incentives for production infrastructure improvements;

- establish procurement models designed to prevent medicine shortages.

The report concludes by stating that research on the topic has been challenging owing to key information gaps in data on shortages, impact on patients and causes of shortages as well as insufficient awareness and accountability.

\section{MIDTERM REVIEW OF THE DIGITAL SINGLE MARKET STRATEGY}

The European Commission recently published a midterm review of the digital single market strategy. The review examines what has been achieved and outlines further actions needed in the key areas cybersecurity, data economy and online platforms.

The document also deals with the transformation of health and care. It emphasises the impact that digital health technologies have on citizens' health, particularly highlighting advantages such as improving access to care and contributing to the sustainability and resilience of healthcare systems. Further advancement of initiatives in the health sector is, however, necessary. This includes supporting the development of a secure data infrastructure to pool health data for further research, disease prevention and personalised health and care; fostering European cooperation to accelerate the large-scale adoption of innovative solutions such as telemedicine and mobile health applications; and making it possible for citizens to access and share their personal health data. It will be ensured that such developments respect data protection rules.

The European Commission is working with Member States on the introduction of systems allowing citizens to transfer their basic medical information electronically when receiving treatment in another Member State and promoting the use of e-prescriptions. Such systems should be operational by 2020. Funding is available for Member States through the Connecting Europe Facility programme.

Moreover, the review indicates that the European Commission is planning to adopt a Communication in 2017 dealing with the need and scope for further measures in the area of digital health and care. The planned publication will, in line with legislation on the protection of personal data, patient rights and electronic identification, address in particular:

- citizens' secure access to electronic health records and the possibility of sharing them across borders and the use of e-prescriptions;

- supporting data infrastructure, to advance research, disease prevention and personalised health and care in key areas, including rare, infectious and complex diseases;

- facilitating feedback and interaction between patients and healthcare providers, to support prevention and citizen empowerment as well as quality and patient-centred care, focusing on chronic diseases and on a better understanding of the outcomes of healthcare systems.

\section{EMA ANNUAL REPORT 2016}

In mid-May, the European Medicines Agency (EMA) released its 2016 annual report. It outlines EMA's key achievements in the evaluation and monitoring of medicines, its strong network for regulatory excellence and its influence on the global regulatory environment.

Advances in the human health sector, as well as contributions to animal and 
human health in relation to veterinary medicines, also played an important role in 2016. These focused on new ways to collect data on medicines such as big data, patient registries and real-world data as well as on EMA's contribution to dealing with public health challenges, including antimicrobial resistance and the Zika virus outbreak. One of the highlights includes the launch of the PRIority MEdicines (PRIME) groundbreaking initiative-supporting the development of medicines that address unmet medical needs-and the decision to publish clinical data, which turned EMA into one of the most transparent medicines regulators worldwide.

Facts and figures presented in the annual report demonstrate the impact of EMA's work, including medicines recommended for approval (81 medicines for human use, including 27 new active substances; 11 medicines for veterinary use, including 6 new active substances), updates of medicine product information on the basis of new safety data (300 medicines for human use) and outcomes of the signal assessment $(94$ confirmed signals prioritised and assessed by the Pharmacovigilance Risk Assessment Committee).

The report also contains three interviews with stakeholders and EMA representatives centring on vaccines, antimicrobial consumption and the development of the EMA, which are topics of major interest in 2016.

\section{NEW DEVELOPMENTS IN BIOSIMILAR MEDICINES}

On 5 May 2017 the European Commission and the EMA published an information guide for healthcare professionals on biosimilar medicines. The guide was developed in collaboration with EU scientific experts, in response to requests from healthcare professionals.

Since the approval of the first biosimilar medicine in 2006, EMA's Committee for Medicinal Products for Human Use has recommended 28 biosimilars for use in the EU. The evidence acquired over 10 years of clinical experience shows that biosimilars approved through EMA can be used as safely and effectively in all their approved indications as other biological medicines.

The growing biosimilar market led in 2013 to the development of a consensus document containing valuable information on biosimilar medicinal products. Further information was provided to patients in 2016, when the European Commission published a Q\&A document addressing the most common questions on biosimilar medicines.

With the information guide for healthcare professionals, EMA and the Commission have now made available a comprehensive set of reliable information on biosimilar medicines. The guide takes into consideration views of organisations from across the EU, representing doctors, nurses, pharmacists and patients who contributed to the drafting process, to ensure that the guide adequately deals with questions relevant to healthcare professionals.

The guide was presented to the public at the third stakeholder conference on biosimilar medicine, organised by the European Commission Directorate-General Internal Market, Industry, Entrepreneurship and SMEs (DG GROW). The event provided patients, doctors, pharmacists, academics, authorities and industry with the opportunity to share their experiences with biosimilar medicines across Europe. In addition, it launched the updated report on the impact of biosimilar competition on price, volume and market share, prepared at the request of the European Commission by QuintilesIMS in cooperation with industry stakeholders.

The updated report describes the effects on price, volume and market share following the arrival and presence of biosimilar competition in the European Economic Area. A set of key performance indicators was used to monitor the impact of biosimilars in European markets in 2016. Evidence presented in the updated report shows a consistent average price reduction in areas where biosimilars have been introduced.

\section{UPDATED TOOLS AVAILABLE FOR SURVEILLANCE OF HEALTHCARE- ASSOCIATED INFECTIONS}

The Healthcare-associated Infections Surveillance Network (HAI-Net), which is coordinated by the European Centre for Disease Prevention and Control (ECDC), released two updated protocols for the surveillance of healthcare-associated infections (HAIs).

One update deals with surgical site infections (SSIs), which are one of the most common HAIs, leading to longer postoperative hospital stays, additional surgical procedures and treatment in an intensive care unit (ICU). The second updated protocol covers HAIs and prevention indicators in ICUs. Patients admitted to ICUs are more at risk of contracting a HAI owing to both intrinsic (eg, immune system depression) and extrinsic (eg, mechanical ventilation) factors. They are also often exposed to antimicrobial resistance problems, which complicate the treatment of severe infections as treatments for these HAIs are limited.

The updates mainly aim at ensuring the standardisation of definitions, data collection and reporting procedures throughout Europe. By aligning definitions and procedures, the prevention and control of HAIs can be increased and the long-term goal of improving the quality of care in European hospitals can be achieved.

The protocols are used by hospitals that are voluntarily participating in the national/regional surveillance of HAIs. An additional objective of the updated HAI-Net SSI and ICU protocols is to compare and follow-up the implementation of key measures for the prevention of SSIs and HAIs in ICUs (especially device-associated pneumonia and bloodstream infections).

Besides the protocols, the HAI-Net also made available a new version of the free surveillance software application. The update now includes the HAI-Net ICU and HAI-Net Clostridium difficile infection modules. It is planned to release the HAI-Net SSI module by the end of this year. Through this surveillance software, data on HAIs are reported by participating hospitals to the national surveillance centres that are part of HAI-Net.

\section{NEW FIGURES ON ANTIBIOTIC CONSUIMPTION IN EASTERN EUROPE}

The WHO Europe region made available a report containing data on consumption of antimicrobial medicines collected from 11 non-European Union countries in the WHO European region and Kosovo. The data were gathered through the WHO Antimicrobial Medicines Consumption (AMC) Network, which was set up in 2011 to assist non-European Union member states to establish or strengthen national surveillance. Albania, Armenia, Azerbaijan, Belarus, Kyrgyzstan, Montenegro, the Republic of Moldova, Serbia, Tajikistan, Turkey and Uzbekistan, as well as Kosovo, are part of the AMC Network.

The report provides insight into the types of antibiotics used within the category of antibacterial agents for systemic use. $\beta$-Lactam antibiotics have been identified as the most commonly consumed subgroup of antibacterial agents. The report also shows that the second-line antibiotics, cephalosporins and quinolones, are consumed in high volumes by some of the surveyed countries. Countries also reported high levels of 
consumption of injectable formulations of antimicrobial agents.

The data gathered within the report indicate that antibiotic consumption varies greatly across the surveyed countries, but the reasons for this are not fully known. Further investigation is necessary, and would also offer opportunities to develop interventions to promote more responsible use of antimicrobial agents.

The report feeds into the global action plan on antimicrobial resistance adopted by WHO member states in 2015 and into the European strategic action plan on antibiotic resistance, which identified monitoring of the use of medicines as a priority for $\mathrm{WHO}$ /Europe.

\section{POTENTIAL FOR EHEALTH TO INCREA VACCINATION COVERAGE}

European Immunisation Week, which took place between 24 and 30 April 2017, was the perfect opportunity for ECDC to release its report on immunisation information systems in the EU and European Economic Area (EEA).

The report provides information on immunisation information systems (IIS) in the EU/EEA countries and describes their functionality. Its promotion coordinated with the objective of the European Immunisation Week to increase awareness of the importance of immunisation. The publication is particularly important for those countries without systems and those in the early stages of implementation of, or updating, their systems.

The report summarises the results of two surveys that ECDC conducted on IIS in the EU/EEA countries. The research focuses, on the one hand, on countries with an established IIS and, on the other, on countries in an earlier stage of IIS implementation. Fourteen countries reported that they had a national system that digitally records information about vaccination, while in seven others, innovative systems are being piloted.

Many of the IIS in operation or being set up make possible the recording of wholeof-life vaccination data, with a small number being able to print a personal immunisation history. Automated reminders are also used by five IIS. These systems can produce lists that identify undervaccinated populations, determine which vaccines are due or overdue, and generate reminders for providers and vaccine recipients.

The importance of digital immunisation records is also recognised by the 2014 EU Council conclusions on vaccination as an effective tool in public health and further endorsed by the WHO's European Vaccines Action Plan.

Overall, the report shows that strong information systems are an integral part of a well-functioning vaccination programme that enables individuals to stay up-to-date with their immunisations throughout their lives and removes uncertainties over vaccination statuses.

\section{NEW EU RULES FOR MEDICAL AND IN VITRO DIAGNOSTIC DEVICES APPROVED}

Medical devices include a wide array of products, ranging from simple plasters to prostheses or pacemakers. They play a central role in maintaining health and quality of life of the population. However, unlike medicinal substances, medical devices do not need premarket authorisation. Instead, medium- and high-risk devices need to pass a conformity assessment, performed by an independent third party, called a 'notified body'.

EU legislation which regulated these devices, approved in the 1990s, was no longer in line with the latest technological advancements. In addition, diverging interpretation of the existing directive by member states and the traceability of the products was problematic.

Therefore, in 2012, the European Commission decided to deal with these concerns by modifying the existing laws. As a first step a public consultation was launched, based on which, amendments to the current legislation should be determined. To ensure a uniform application throughout the EU, it was decided to replace the existing directives on medical devices and in vitro diagnostic devices by regulations.

The two sets of regulations, approved by the European Parliament focus on the following aspects:

- widening and clarifying the scope of the legislation, including for example, aesthetic implants and medical software;

- ensuring a closer supervision of the notified bodies by the national authority;

- offering more responsibility and authority to the assessment bodies, including regular checks on manufacturers and thorough testing;

- clarifying the rights and obligations of the parties in the supply chain, covering also internet sales and diagnostic services;

- ensuring transparency for patients and healthcare professionals through a database (EUDAMED) containing comprehensive information about the devices available on the EU Market;

- introducing a unique device identification system (UDI system) to ensure postmarketing surveillance and traceability;

- implementing stricter requirements for clinical evidence;

- adapting the safety and performance requirements to new health technologies;

- enhancing coordination between national surveillance authorities and aligning with international guidelines to facilitate trade.

The next step is the implementation of the legislation, which would be a gradual process, ranging from 3 years for medical devices to 5 years for in vitro diagnostic devices. Directorate-General Internal Market, Industry, Entrepreneurship and SMEs (DG GROW) is in charge of implementing the UDI system. This task will be carried out by an EU expert group on UDI that will assist in preparing regulatory acts/guidance related to UDI implementation.

Both Regulation (EU) 2017/745 of the European Parliament and of the Council of 5 April 2017 on medical devices, amending Directive 2001/83/ EC, Regulation (EC) No 178/2002 and Regulation (EC) No 1223/2009 and repealing Council Directives 90/385/ EEC and 93/42/EEC and Regulation (EU) 2017/746 of the European Parliament and of the Council of 5 April 2017 on in vitro diagnostic medical devices and repealing Directive 98/79/EC and Commission Decision 2010/227/EU were published in the official journal of the European Union on 5 May 2017.

\section{WHO LAUNCHES GLOBAL PATIENT SAFETY CHALLENGE ON MEDICATION SAFETY}

WHO has launched a new global initiative-the Global Patient Safety Challenge on Medication Safety-seeking to halve medication-related errors over the next 5 years.

Mistakes in ordering, prescribing, dispensing, preparing, administering or consuming medicine can be made by both health workers and patients. Such mistakes could be avoided, however, through systems and procedures which ensure that the right patient receives the right medication at the right dose via the right route at the right time. Thus, the $\mathrm{WHO}$ initiative calls on countries to take priority action in dealing with system failures in the way care is organised and coordinated. 
Actions taken as part of the Global Patient Safety Challenge on Medication Safety will focus on patients and the public, healthcare professionals, medicines as products, and systems and practices of medication.

The Global Patient Safety Challenge on Medication Safety seeks to improve the way in which medicines are prescribed, distributed and consumed, and to increase awareness among patients about the risks associated with the improper use of medication.
EAHP Statement 6: Education and

Research

Competing interests None declared.

Provenance and peer review Commissioned; internally peer reviewed.

(C) European Association of Hospital Pharmacists (unless otherwise stated in the text of the article) 2017.

All rights reserved. No commercial use is permitted unless otherwise expressly granted.
To cite Kohl S. Eur J Hosp Pharm 2017;24:248-251.

Received 1 June 2017

Accepted 1 June 2017

Accepted 1 June 2017

Eur J Hosp Pharm 2017;24:248-251. doi:10.1136/ejhpharm-2017-001321 